\title{
The associative learning of the deaf: The effects of word imagery and signability
}

\author{
DONNA CONLIN and ALLAN PAIVIO \\ University of Western Ontario, London 72, Ontario, Canada
}

\begin{abstract}
In two experiments, deaf and hearing subjects learned paired associate lists in which rated visual imagery and signability (a measure of the ease with which a word can be represented as a gestural sign) were orthogonally varied. Visual presentation of three alternating study-recall trials resulted in significant positive effects of imagery for both deaf and hearing subjects, whereas signability facilitated recall only for deaf subjects. Examination of the relation between item attributes and reported learning strategy indicated that both deaf and hearing subjects used imaginal mediators more frequently for high-imagery than low-imagery pairs. A gestural sign strategy was reported almost exclusively by deaf subjects, particularly for high-signability pairs. These results suggest that an examination of the effects of sign language variables will contribute to an understanding of the qualitative differences in the associative learning of the deaf and hearing.
\end{abstract}

Paivio and Okovita (1971) demonstrated that the word attribute of imagery concreteness is modality specific. They found that congenitally blind subjects learned word pairs with high auditory imagery (that is, with auditory referents) more easily than pairs low in auditory imagery; however, high visual imagery was not similarly helpful. On the other hand, the associative recall performance of sighted subjects was positively affected by visual but not by auditory word imagery. This finding is consistent with the view that different groups of subjects avail themselves of different coding strategies. Not only may the effectiveness of a particular word attribute vary somewhat with the subject population being considered, but also particular attributes may be relevant to one group and not to another.

The word attribute of signability apparently is an instance of the latter type. In American sign language (ASL), some words must be fingerspelled; others are conveyed by relatively simple manual configurations or gestures; still others are related, either semantically or syntactically, to words that can be signed directly. The word as spoken either has or does not have an ASL equivalent for a given individual; in this sense, word signability is an index of the ease with which words can be represented as gestural signs. The extent and, to a certain degree, the topology of the sign vocabulary varies across individuals; accordingly, the signability dimension assesses commonality of response. In summary, three points concerning the signability dimension can be made: A word high in rated signability is easily

Based in part on research completed in partial fulfillment of the requirement for the MA degree by the first author under the direction of the second author. The research was supported in part by grants from the National Research Council of Canada (APA-0087). We are grateful for the cooperation given us by the staff and students at the Ontario School for the Deaf (Milton) Central Secondary School (London), and the National Technical Institute for the Deaf (Rochester). A special thanks is extended to Mr. Etherington of OSD and Ms. Frishberg of NTID, who served as signers in the experiments. translated into a sign equivalent by (almost) all ASL users, whereas few or none have sign equivalents for low-signability words; and, for words rated somewhere between the extremes, only some persons have signs in their gestural repertoire, or most persons have the signs but experience difficulty in rendering the appropriate meanings via signs.

An experiment reported by Odom, Blanton, and McIntyre (1970) suggests that word signability may be a critical variable in the verbal learning performance of the deaf. They found that deaf, but not hearing, subjects learned significantly more words that had sign equivalents than words that did not; thus, an item's signability seemed to be an important determinant of whether or not that item was recalled by deaf subjects. However, several important word attributes such as part of speech and word imagery-concreteness were not controlled, and alternative interpretations of the results are accordingly possible.

The two experiments reported here attempted to further our understanding of the role of gestural signs as mediators of verbal learning. Both involved systematic variation in the rated signability and visual imagery value of words in a paired associate (PA) learning situation. Three predictions were made with respect to the PA task. Since visual experience is of primary importance in the lives of both deaf and nonsensorily deprived persons, it was hypothesized that the performance of deaf subjects would be qualitatively equivalent to that of hearing subjects on the visual imagery dimension; that is, both groups should recall more high-visual than low-visual pairs. On the other hand, variations in the signability dimension should affect only deaf subjects' recall: The deaf, but not the hearing (for whom the dimension is irrelevant), should recall more high-signable than low-signable pairs. Previous research (e.g., Dalton, 1969) also led us to expect that the performance of the deaf subjects would be generally inferior to that of the 
hearing. The suggestion that the deaf use different learning strategies than do the hearing (Allen, 1970, 1971; Blanton \& Nunnally, 1967; Conrad \& Rush, 1965) was also examined by means of a postlearning questionnaire in which subjects indicated the type of mnemonic strategy they had employed during the PA task; of particular interest was the possibility that deaf subjects would report frequent use of gestural mediation, particularly for high-signable pairs.

\section{EXPERIMENT I}

\section{Method}

Paired associate list. Forty experimental words were selected from a pool of 600 nouns (Conlin, 1972) which had been rated in terms of image-evoking capacity and in terms of the ease with which a word evoked a sign equivalent; for both dimensions, the procedure followed that used by Paivio, Yuille, and Madigan (1968). Twenty words were high and 20 low in visual imagery; within each imagery level, half of the nouns were high in rated signability, half were low. Thus, there were four categories of words: low imagery (I) and low signability (S), low I and high S, high $I$ and low $S$, and high $I$ and high $S$. Observing these categorical boundaries, two random pairings of the nouns were produced, yielding two equivalent PA tests of 20 noun pairs. Within each list, five of the pairs were constructed from low-I/low-S words, five from low-I/high-S words, five from high-I/low-S words, and five from high-I/high-S words. The mean normative values for visual imagery and signability, respectively, are 3.65 and 2.07 for the low-1/low-S pairs, 3.71 and 6.33 for the low-I/high-S pairs, 6.30 and 2.23 for the high-I/low-S pairs, and 6.35 and 6.37 for the high-I/high-S pairs. Between categories, the items were equated on frequency, according to the Kucera-Francis (1967) word count.

Subjects. Thirty prelingually deaf students (average hearing loss $=65 \mathrm{~dB}, \mathrm{ASA}$ ) from the Ontario School for the Deaf (Milton) and 30 hearing controls from Central Secondary School (London) served as subjects. The two groups were equivalent in terms of mean age (deaf $=17.0$ years, hearing $=16.4$ years) and average IQ (deaf $=104.9$, hearing $=107.9$ ); there was an equal number of males and females in each group.

Procedure. Tested in groups of seven or eight, subjects were given printed instructions describing the PA task, followed by one practice trial with three extra pairs; to insure that the deaf subjects understood the instructions, a signer was present at their sessions. The subjects were given three alternating study trials (presentation of pairs) and test trials (presentation of the first member of each pair with subjects attempting to recall the second member) with the complete experimental list. The items were presented visually on $20 \times 5$ in. flashcards, at a 4-sec rate on study trials and an 8-sec rate on test trials; a different random ordering of the items was used on each trial. In each group, 15 subjects were tested with one random pairing of the nouns, whereas the remaining subjects received the other randomly paired list. In addition, pair members were reversed for different subgroups; for example, one group received "mercy-triumph," another "lecture-mercy," a third "triumph-mercy," and a fourth "mercy-lecture." Subjects responded in writing on test trials.

Following the PA task, subjects were required to complete a strategy questionnaire which described five devices or tricks by which subjects may have learned the members of the list, namely, sign or gesture, verbal associate, mental image, repetition, and other strategies. The sign strategy was of particular interest due to its direct connection with the communication system of the deaf; it was reasoned that deaf subjects might remember the sign for one or both members of a pair and use this information as a learning aid. The verbal strategy involved using a word, phrase, or sentence to connect the members of a pair, while the image strategy involved picturing the meanings of the words. The final alternatives involved either rote repetition of the pair or some idiosyncratic, unclassified learning aid. Subjects were directed to consider each noun pair individually when indicating which strategy (one response per pair) they used.

\section{Results and Discussion}

Paired associate recall. Recall scores were determined for each subject for each pair category on each trial; the means for these scores are presented in Table 1. Analysis by a 2 (groups) by 3 (trials) by 2 (signability) analysis of variance (the last three factors involving repeated measures) revealed significant effects attributable to each of the four factors. As expected, the recall performance of the hearing $(\overline{\mathrm{X}}=36.6)$ was superior to that of the deaf $(\bar{X}=24.3)[F(1,58)=21.9, p<.01]$ and recall increased over trials $[F(2,116)=305.2$, $p<.01]$. The effects involving item attributes are more interesting here. Recall was generally better for high-imagery than for low-imagery pairs $[F(1,58)=$ $158.3, \mathrm{p}<.01]$ and this effect was only somewhat qualified by interactions. For both groups, the facilitating effect of imagery was greater on later than earlier trials $[F(2,116)=8.13, p<.01]$. This was further qualified by a triple interaction of Groups by Trials by Imagery $[F(2,116)=3.71, p<.01]$, which perhaps reflects a ceiling effect such that the high-I/low-I recall difference decreased over trials for hearing subjects. In any case, the prediction that both groups would perform comparably with respect to the imagery dimension was essentially confirmed.

Recall of high-S pairs exceeded recall of low-S pairs $[F(1,58)=25.26, p<.01]$. This main effect was qualified by a Groups by Signability interaction $[F(1,58)=8.27, p<.01]$, which confirms the expectation that the facilitative effect of signability is restricted to deaf subjects. In addition, the significant Imagery by Signability interaction $[F(1,58)=24.11$, $p<.01]$ indicates that the effect of signability depends on the level of imagery, with signability facilitating recall when pairs are high but not when they are low in imagery.

Table 1

Mean Correct Recall (Maximum Per Cell $=5.0$ )

\begin{tabular}{|c|c|c|c|c|c|c|c|}
\hline & \multicolumn{2}{|c|}{ Trial 1} & \multicolumn{2}{|c|}{ Trial 2} & \multicolumn{2}{|c|}{ Trial 3} \\
\hline & & M & SD & M & SD & M & SD \\
\hline \multirow{4}{*}{ Deaf } & Low I/Low $S$ & .40 & .71 & 1.07 & 1.06 & 2.27 & 1.65 \\
\hline & Low I/High S & .53 & .72 & 1.43 & 1.12 & 2.43 & 1.43 \\
\hline & High I/Low S & .67 & .98 & 2.07 & 1.44 & 3.60 & 1.53 \\
\hline & High $\mathbf{I} /$ High S & 1.83 & 1.27 & 3.57 & 1.51 & 4.43 & .97 \\
\hline \multirow{4}{*}{$\begin{array}{l}\text { Hear- } \\
\text { ing }\end{array}$} & Low I/Lov & 1.23 & 1.02 & 2.47 & 1.31 & 3.70 & 1.14 \\
\hline & Low I/High & 1.37 & 1.17 & 2.60 & 1.33 & 3.40 & 1.36 \\
\hline & High $\mathrm{I} /$ Low S & 1.97 & 1.40 & 3.77 & 1.42 & 4.60 & .66 \\
\hline & High $1 /$ High S & 2.70 & 1.46 & 4.13 & .91 & 4.67 & .65 \\
\hline
\end{tabular}


Mediation reports. Analyses of the mediation reports were concerned with the relation of frequency of reported use of a strategy to attributes of the noun pairs, type of strategy, and subject type. The reports were scored by summing the number of pairs of each word type for which a given strategy was reported for each subject; the reported frequency of a given strategy could range from 0 to 5 for a particular word type. These data are summarized in Table 2 . The results were analyzed by a 2 by 5 by 2 by 2 analysis of variance, with groups, strategies, imagery, and signability as factors, the last three involving repeated measures. Of the four factors, only strategies emerged as a main effect $[F(4,232)=$ $17.47, \mathrm{p}<.01]$. In addition, the reported usage of different strategies was affected by pair attribute and hearing status: Both the Strategies by Imagery interaction $[F(4,232)=23.56, p<.01]$ and Groups by Strategies interaction $[F(4,232)=3.48, p<.01]$ were significant. In order to interpret these effects, separate three-factor repeated-measures analyses of variance were performed on the sign, verbal, image, and repetition mediation data; since additional information was lacking with respect to the "other" strategy, it was excluded from further analysis.

The use of gestures or signs was significantly higher for deaf than for hearing subjects $[F(1,58)=17.16$, $p<.01]$. This finding is in accord with the general consensus that the deaf bring different, sometimes unique, approaches to the verbal learning situation. That the sign strategy was reported at all by hearing subjects is puzzling, since these subjects were unfamiliar with ASL; it may be that the three hearing subjects who reported using sign mediators misunderstood what constitutes a sign strategy and were, in fact, using some other mediator such as shape or movement. The significant Groups by Signability interaction $[\mathrm{F}(1,58)=$ $8.371, \mathrm{p}<.01]$ is particularly important from a theoretical viewpoint. Deaf subjects reported the sign strategy significantly more often for high-S as compared to low-S pairs, whereas the hearing subjects showed no such differences.
A number of investigators (e.g., Furth, 1966; Moores, 1970) have asserted that deaf subjects typically experience difficulty in the input and output of phrases or sentences. In the present study, however, the overall incidence of verbal mediation did not differ significantly $(\mathrm{F}=.579)$ among deaf and hearing subjects. The contention that the deaf prefer strategies other than verbal is nonetheless warranted when all the mediation data are considered. Deaf subjects expressed a preference for the repetition strategy, which may explain their inferior recall performance. The nature of this repetition strategy merits further study. Observation of the deaf subjects during learning suggests that they do not subvocally repeat material; rather, they use explicit motor signs.

Finally, reported usage of the imagery strategy differed significantly between groups $[F(1,58)=6.14$, $p<.05]$, with the hearing subjects reporting the strategy more often. Both deaf and hearing subjects reported using imagery more often with high-I than with low-I pairs $[F(1,58)=35.281, p<.01]$. In summary, the mediation reports reveal both striking parallels and interesting differences in the strategies which the deaf and the hearing bring to the verbal learning situation.

\section{EXPERIMENT II}

The second experiment was designed to test the generality of the effects obtained in Experiment I. It differed from the first in two respects. First, different groups of subjects received entirely different word lists, thus implementing Clark's (1973) suggestions regarding the "language as fixed effect fallacy." Second, subjects were all college students. Often comparisons between the deaf and hearing are hampered by the difficulty in obtaining appropriate matched comparisons groups; matching on the basis of age alone, (nonverbal) IQ alone, or reading level alone is not sufficient. Use of college populations should largely circumvent these restrictions. Accordingly, correspondences in the results of the two experiments will indicate the generality of the effects

Table 2

Mean Number of Pairs on Which Particular Mediators Were Reported as a Function of Imagery and Signability

\begin{tabular}{|c|c|c|c|c|c|c|c|c|c|c|c|}
\hline & & \multicolumn{2}{|c|}{ Sign } & \multicolumn{2}{|c|}{ Verbal } & \multicolumn{2}{|c|}{$\begin{array}{l}\text { Reported Mediator } \\
\text { Image }\end{array}$} & \multicolumn{2}{|c|}{ Repetition } & \multicolumn{2}{|c|}{ Other } \\
\hline & & M & $\mathrm{SD}$ & $\mathbf{M}$ & $\mathrm{SD}$ & M & SD & M & SD & $\mathbf{M}$ & SD \\
\hline \multirow{4}{*}{ Deaf } & Low I/Low S & .47 & .88 & .67 & .94 & .60 & 1.05 & 2.10 & 1.79 & 1.17 & 1.27 \\
\hline & Low I/High S & .80 & 1.17 & .60 & .84 & .70 & .86 & 2.07 & 1.53 & .83 & 1.13 \\
\hline & High I/Low S & .60 & 1.02 & 1.03 & 1.11 & 1.70 & 1.39 & 1.27 & 1.37 & .40 & .66 \\
\hline & High I/High S & 1.30 & 1.49 & 1.00 & 1.15 & 1.67 & 1.59 & .87 & 1.47 & .13 & .43 \\
\hline \multirow{4}{*}{ Hearing } & Low $\mathrm{I} /$ Low $\mathrm{S}$ & .03 & .18 & 1.07 & 1.44 & 1.27 & 1.09 & 1.97 & 1.35 & .70 & .90 \\
\hline & Low I/High S & .10 & .30 & 1.37 & 1.72 & .97 & 1.13 & 2.33 & 1.66 & .33 & .65 \\
\hline & High $\mathrm{I} /$ Low $\mathrm{S}$ & .20 & .48 & .80 & 1.28 & 2.37 & 1.65 & 1.50 & 1.57 & .10 & .30 \\
\hline & High $\mathrm{I} /$ High $\mathrm{S}$ & .20 & .65 & .77 & 1.23 & 2.53 & 1.60 & .90 & 1.13 & .53 & .92 \\
\hline
\end{tabular}


across different groups and different learning items.

\section{Method}

Subjects. Twenty-six deaf students from the National Technical Institute for the Deaf in Rochester (mean age $=21.9$ years) and 26 hearing controls from the University of Western Ontario (mean age $=19.5$ years) served as subjects. The deaf subjects each received $\$ 3$ for their participation, while the hearing subjects were freshmen fulfilling an introductory course requirement. Within each group there were 14 males and 12 females; in addition, within the deaf group, 13 subjects had used ASL for several years prior to their entrance to NTID, while the others had only subsequently acquired ASL. This experiential difference had no effect on the results of the study and it will not be considered further.

Paired associate lists. Two 16-pair PA lists were constructed such that each contained four low-I/low-S pairs, four low-I/high-S pairs, four high-I/low-S pairs, and four high-I/high-S pairs. The mean normative values for visual imagery and signability, respectively, are 2.65 and 2.23 for low-I/low-S pairs, 3.11 and 6.09 for low-I/high-S pairs, 6.00 and 2.56 for high-I/low-S pairs, and 6.51 and 6.11 for high-I/high-S pairs. Between categories, the items were equated on mean frequency, according to the Kučera-Francis (1967) word count.

Procedure. Tested in small groups, subjects were given standard PA learning instructions followed by three alternating study-recall trials. Fourteen deaf and 14 hearing subjects were tested with one list, whereas the remaining subjects received the second list; in addition, the left-right position of pair members was reversed for different subgroups. After PA learning, subjects completed the same five-alternative strategy questionnaire as in Experiment I; additional information relevant to the other/idiosyncratic strategy was obtained from an open-ended questionnaire in which subjects were asked to describe the strategy.

\section{Results and Discussion}

Paired associate recall. The PA data, summarized in Table 3 , were analyzed by a 2 by 2 by 3 by 2 by 2 analysis of variance, with groups, lists, trials, imagery, and signability as factors. Lists was a random factor, while the others were fixed. The analysis revealed no main effect $[F(1,48)=.19]$ attributable to the lists factor; in addition, the factor entered into no significant interactions involving subject type or pair attribute. The minimal variation contributed by this factor resulted in what were considered inflated estimates of the other effects of theoretical interest here; accordingly, a four-factor analysis of variance was performed. This analysis in no way altered the pattern of results but did yield more conservative estimates of the effects. It is the results of this analysis which are reported below.

Significant effects were attributable to each of the four factors. The recall performance of the hearing $(\bar{X}=33.58)$ was again superior to that of the deaf subjects $(\overline{\mathrm{X}}=28.04) \quad[\mathrm{F}(1,50)=4.97, \mathrm{p}<.05]$, and recall scores increased across trials $[F(2,100)=220.00$, $p<.011$. Compared to the other level of each factor, recall was better for pairs in which nouns were high I $[F(1,50)=18.05, p<.01]$ and for high-S pairs $[F(1,50)$ $=25.67, \mathrm{p}<.01]$.

The main effects were qualified by several significant
Table 3

Mean Correct Recall (Maximum Per Cell $=4.0$ )

\begin{tabular}{|c|c|c|c|c|c|c|c|}
\hline & & \multicolumn{2}{|c|}{ Trial 1} & \multicolumn{2}{|c|}{ Trial 2} & \multicolumn{2}{|c|}{ Trial 3} \\
\hline & & $\mathbf{M}$ & SD & $\mathbf{M}$ & SD & $\mathbf{M}$ & SD \\
\hline \multirow{4}{*}{ Deaf } & Low $\mathrm{I} /$ Low $\mathrm{S}$ & .89 & .85 & 1.85 & 1.26 & 2.73 & 1.38 \\
\hline & Low I/High S & 1.54 & 1.19 & 2.85 & 1.17 & 3.27 & .96 \\
\hline & High I/Low S & 1.23 & .89 & 2.19 & 1.21 & 3.08 & 1.06 \\
\hline & High I/High S & 1.46 & 1.36 & 3.31 & .75 & 3.65 & .59 \\
\hline \multirow{4}{*}{$\begin{array}{l}\text { Hear- } \\
\text { ing }\end{array}$} & Low I/Low S & 1.35 & 1.03 & 2.85 & 1.29 & 3.65 & .99 \\
\hline & Low $/ /$ High S & 1.85 & 1.18 & 2.69 & 1.29 & 3.31 & .93 \\
\hline & High I/Low S & 1.85 & 1.17 & 3.31 & .87 & 3.73 & .53 \\
\hline & High I/High S & 1.81 & 1.24 & 3.35 & .89 & 3.85 & .46 \\
\hline
\end{tabular}

interactions of theoretical interest. A Groups by Signability interaction $[F(1,50)=22.94, p<.01]$ again indicates that the facilitative effect of signability was restricted to deaf subjects. Scheffé comparisons [mean square error $(\mathrm{MSE})=.756$, critical value $(\mathrm{CV})=7.17]$ verified that the high-S/low-S difference is significant only for deaf subjects $[F(1,50)=20.84, p<.01]$. This effect was somewhat qualified by a triple interaction involving trials $[\mathrm{F}(2,100)=5.88, \mathrm{p}<.01]$. For the deaf, Scheffé (.01) confidence intervals (MSE $=.451$, $\mathrm{CV}=6.90$ ) indicated that recall of high-S pairs exceeded recall of low-S pairs on all trials, whereas, for the hearing, high-S recall exceeded low-S recall only on Trial $1[\mathrm{~F}(1,100)=5.92, \mathrm{p}<.05]$.

Except for the absence of a significant Imagery by Signability interaction, these findings closely parallel those obtained in Experiment I. Thus, it can be concluded that variations in the signability dimension affect only the deaf, whereas variations in the imagery dimension affect both the deaf and the hearing.

Mediation reports. Table 4 summarizes the mediation data (which were scored as in Experiment I). The analysis again indicated that reported usage of different strategies was affected by imagery level $[F(4,200)=$ $6.40, p<.01]$ and hearing status $[F(4,200)=10.60$, $p<.01]$. Separate repeated-measures analyses of variance for the sign, verbal, image, repetition, and idiosyncratic mediation data were performed to better interpret these effects.

The analysis of the sign mediation data replicated the results of Experiment I: Both the main effect involving groups $[F(1,50)=9.384, p<.01]$ and the Groups by Signability interaction $[F(1,80)=6.107, p<.05]$ were significant. Only one hearing subject reported using signs, whereas more than half of the deaf subjects reported such mediators, particularly for high-S pairs $[F(1,50)=10.22, \quad p<.01 \quad$ (Scheffé procedure, $\mathrm{MSE}=.177, \mathrm{CV}=7.17)]$.

Across word types, hearing subjects reported more overall use of verbal mediation than did the deaf $[F(1,80)=11.67, p<.01]$. The absence of any Group by Word Attribute interactions indicates that the two 
Table 4

Mean Number of Pairs on Which Particular Mediators Were Reported

\begin{tabular}{|c|c|c|c|c|c|c|c|c|c|c|c|}
\hline & & \multicolumn{2}{|c|}{ Sign } & \multicolumn{2}{|c|}{ Verbal } & \multicolumn{2}{|c|}{$\begin{array}{l}\text { Reported Mediator } \\
\text { Image }\end{array}$} & \multicolumn{2}{|c|}{ Repetition } & \multicolumn{2}{|c|}{ Other } \\
\hline & & $\mathbf{M}$ & SD & $\mathbf{M}$ & SD & M & SD & M & SD & $\mathbf{M}$ & SD \\
\hline Deaf & $\begin{array}{l}\text { Low I/Low S } \\
\text { Low I/High S } \\
\text { High I/Low S } \\
\text { High I/High S }\end{array}$ & $\begin{array}{l}.19 \\
.69 \\
.46 \\
.50\end{array}$ & $\begin{array}{r}.48 \\
1.07 \\
.97 \\
.89\end{array}$ & $\begin{array}{l}.50 \\
.96 \\
.35 \\
.65\end{array}$ & $\begin{array}{l}.69 \\
.96 \\
.62 \\
.68\end{array}$ & $\begin{array}{r}.62 \\
.54 \\
.62 \\
1.23\end{array}$ & $\begin{array}{l}.74 \\
.75 \\
.68 \\
.97\end{array}$ & $\begin{array}{r}1.77 \\
.89 \\
1.58 \\
.65\end{array}$ & $\begin{array}{r}1.45 \\
.97 \\
1.28 \\
.99\end{array}$ & $\begin{array}{r}.92 \\
.96 \\
1.00 \\
.96\end{array}$ & $\begin{array}{l}1.03 \\
1.22 \\
1.24 \\
1.22\end{array}$ \\
\hline Hearing & $\begin{array}{l}\text { Low } 1 / \text { Low } S \\
\text { Low } 1 / \text { High S } \\
\text { High } 1 / \text { High S } \\
\text { High } 1 / \text { High S }\end{array}$ & $\begin{array}{l}.04 \\
.00 \\
.00 \\
.00 \\
\end{array}$ & $\begin{array}{l}.19 \\
.00 \\
.00 \\
.00 \\
\end{array}$ & $\begin{array}{l}1.19 \\
1.54 \\
1.00 \\
1.50\end{array}$ & $\begin{array}{l}1.18 \\
1.19 \\
1.07 \\
1.28 \\
\end{array}$ & $\begin{array}{r}.65 \\
.42 \\
1.12 \\
1.19 \\
\end{array}$ & $\begin{array}{l}.96 \\
.57 \\
.89 \\
.88 \\
\end{array}$ & $\begin{array}{r}1.62 \\
1.50 \\
1.42 \\
.85 \\
\end{array}$ & $\begin{array}{l}1.08 \\
1.12 \\
1.05 \\
1.06\end{array}$ & $\begin{array}{l}.50 \\
.54 \\
.46 \\
.46 \\
\end{array}$ & $\begin{array}{r}1.01 \\
.79 \\
.63 \\
.69 \\
\end{array}$ \\
\hline
\end{tabular}

groups did not differ in their pattern of usage; subjects in both groups reported the verbal strategy equally often for all word types. On the other hand, word attributes were an important determinant of the frequency with which imaginal and repetition mediators were reported. Both deaf and hearing subjects reported imaginal mediators more frequently for high-I than low-I pairs $[F(1,80)=15.59, p<.01]$. Conversely, the repetition strategy was reported more frequently for low-I as compared to high-I pairs $[F(1,50)=7.72, p<.01]$. Repetition was also reported more often for low-S than high-S pairs $[F(1,50)=20.56, p<.01]$, but this effect was qualified by a Groups by Signability interaction $[F(1,50)=4.09, p<.05]$, which indicated that the low-S/high-S difference was significant only for deaf subjects. Subsequent oral questioning of the deaf subjects suggested that visual rather than auditory-verbal-linguistic factors may be paramount in determining their use of a repetition strategy. Several deaf subjects reported that they visualized the word pairs and read and reread them; this contrasts with hearing subjects' reports of frequent subvocal repetition of to-be-learned items.

The apparent emphasis on visual features and processes by the deaf is substantiated by data involving the "other" strategy. This final alternative again differentiated the two groups, with deaf subjects reporting the strategy more frequently than hearing subjects $[F(1,50)=4.17, p<.05]$. Examination of the open-ended questionnaire revealed that such visual word features as letter commonality (e.g., oats-settler, cane-ink, whale-rattle) may have been the basis of what the deaf subject classified as usage of this strategy; in addition, several reported that they paid particular attention to double letters (e.g., robbery-breeze). In both instances, double occurrences of letters functioned as the critical feature of the mnemonic; this contrasts with hearing subjects' use of phonemic similarities and accentuates the need for a detailed examination of the unique strategies used by the deaf.

\section{GENERAL DISCUSSION}

The major predictions concerning PA performance were confirmed in both experiments. As anticipated, the recall of high-I words surpassed the recall of low-I words among both groups, presumably because both deaf and hearing subjects had previous experience with the visual referents of the concrete nouns and could take advantage of the visual imagery aroused by such nouns during learning. The comparability of the imagery effect for the two groups, as indicated by the absence of a significant Groups by Imagery interaction in either experiment, undercuts any argument of extraordinary compensatory visual skills or storage capacity in the deaf, such as proposed by Blanton and Nunnally (1964). Such compensatory views are questionable on other grounds as well. Clark and Leslie (1971) found that deaf children's performance on visual-motor tasks was generally below that of their hearing peers. Henderson and Henderson (Note 1), using a tachistoscopic letter report task, found that both deaf subjects' introspections and the loss of spatial information without loss of item information contradicted any compensatory theories. Such findings are inconsistent with Craig's (1973) conjecture that, relative to hearing subjects, deaf subjects will demonstrate greater high-I/low-I differences in recall; rather, the reactions of the deaf and the hearing to this variable are comparable, and similar explanations in terms of imagery mediation may be appropriate to both.

The PA results attest to the functional reality of signs for the deaf (cf. Bellugi \& Klima, 1972), although the precise nature of this signability effect was not determined in this research. One possibility is that signs render words more meaningful for the deaf but not for the hearing. These gestural meaning reactions can then function as associative mediators, much as do images or sentences. On the other hand, such mediators are not immediately available for low-S pairs. This differential availability of effective mediators could thus account for 
deaf subjects' superior recall of high-S as compared to low-S pairs.

The mediation reports are relevant to the above discussion as well as being interesting in their own right, although it is obvious that they may not accurately reflect what subjects used to link stimulus and response terms during learning. Three of these strategies, namely, image, sign, and "other," are of particular importance. Although deaf and hearing subjects differed somewhat in the extent of their utilization of the image strategy, both reported imaginal mediators most frequently for high-I pairs (cf. Paivio, Yuille, \& Smythe, 1966). This is consistent with the view that images are more useful in dealing with concrete situations than with abstract (Paivio, 1971). It is possible, of course, that the deaf subjects' usage of the strategy may have involved cognitive manipulations somewhat different from those performed by hearing subjects using what is nominally the same strategy. ${ }^{2}$ For example, deaf subjects might form an image of the relevant sign, whereas hearing subjects form an image of the appropriate external referent. Such a difference, however, should be reflected in a triple interaction of Groups by Imagery by Signability, which was not significant in either experiment. Further, regardless of signability level, both groups reported the image strategy more frequently for high-I than low-I pairs. Thus, the general pattern of usage appears to be the same for both deaf and hearing subjects.

The similarities in the strategies reported by the deaf and hearing are overshadowed by the unique theoretical significance of the sign strategy for the deaf. In both experiments, deaf subjects reported using a gestural sign strategy with high-S words. At this time, it is not known whether gestural mediation (see above) or rehearsal strategy (i.e., an ASL analog of subvocal repetition) is the more appropriate designation for the relation between the word attribute of signability and the reported use of (explicit or implicit) motor signs. Further investigation, perhaps by means of instructional sets, should explicate the relationship. Similarly, the other/idiosyncratic strategy should be comprehensively examined to determine both its critical features and its generality.

The results of these two experiments provide several clues to the nature of the information processing system of the deaf: Gestural signs, visual features (perhaps also intimately related to ASL, the "visual" language of the deaf), and visual images seem to play major roles in their symbolization of verbal material. More generally, these results provide further evidence that alternative encoding modes can be used effectively.

\section{REFERENCES}

Allen, D. V. Acoustic interference in paired-associate learning as a function of hearing ability. Psychonomic Science, 1970, 18 , 231-233.

Allen, D. V. Color-word interference in deaf and hearing children. Psychonomic Science, 1971, 24, 295-296.

Bellugi, U., \& Klima, E. S. The roots of language in the sign talk of the deaf. Psychology Today, June 1972, 61-76.

Blanton, R. L., \& Nunnally, J. C. Semantic habits and cognitive style processes in the deaf. Journal of Abnormal Social Psychology, 1964, 68, 397-402.

Blanton, R. L., \& Nunnally, J. C. Retention of trigrams by deaf and hearing subjects as a function of pronunciability. Journal of Verbal Learning and Verbal Behavior, 1967, 6, 428-431.

Clark, H. H. The language-as-fixed-effect fallacy: A critique of language statistics in psychological research. Journal of Verbal Learning and Verbal Behavior, 1973, 12, 335-359.

Clarke, B. R., \& Leslie, P. T. Visual-motor skills and reading ability of deaf children. Perceptual and Motor Skills, 1973, 33, 263-268.

Conlin, D. R. The effects of word imagery and signability in the paired-assoc iate learning of the deaf. Master's thesis, University of Western Ontario, 1972.

Conrad, R., \& Rush, M. L. On the nature of short-term memory encoding by the deaf. Journal of Speech and Hearing Disorders, 1965, 30, 336-343.

Craig, E. M. The role of mental imagery in the free-recall of deaf blind, and normal subjects. Journal of Experimental Psychology, 1973, 97, 249-259.

Dalton, D. A. Paired-associate learning in children with sensorineural hearing losses. Dissertation Abstracts International, 1969, 30(1), 55-56.

Furth, G. H. Thinking without language. New York: Free Press, 1966.

Kučera, H., \& Francis, W. N. Computational analysis of present-day American English. Providence, R.I: Brown University Press, 1967.

Moores, D. F. Psycholinguistics and deafness. American Annals of the Deaf, 1970, 115, 37-40.

Odom, P. B., Blanton, R. L., \& McIntyre, C. K. Coding medium and word recall by deaf and hearing subjects. Journal of Speech and Hearing Research, 1970, 13, 54-59.

Paivio, A. Imagery and verbal processes. New York: Holt, Rinehart, \& Winston, 1971.

Paivio, A., \& Okovita, H. W. Word imagery modalities and associative learning in blind and sighted subjects. Journal of Verbal Learning and Verbal Behavior, 1971, 10, 506-510.

Paivio, A.. Yuille. J., \& Madigan, S. Concreteness, imagery, and meaningfulness values for 925 nouns. Journal of Experimental Psychology, 1968, 76(1, Pt. 2).

Paivio, A., Yuille, J., \& Smythe, P. C. Stimulus and response abstractness, imagery and meaningfulness, and reported mediators in paired-associate learning. Canadian Journal of Psychology, 1966, 20, 362-377.

\section{NOTES}

1. Henderson, L., and Henderson, S. E. Tachistoscopic report in the deaf and models of short-term memory. Paper presented at the annual meeting of the Canadian Psychological Association, 1972 .

2. The same argument can be made with respect to the repetition strategy. Whereas the hearing subvocally repeat the material, the deaf may either manually or visually (read and reread) rehearse.

(Received for publication July 21, 1974; revision accepted September 14,1974 .) 